\title{
A Case Report: Trans-Thoracic Pigtail Catheter with Diaphragm Perforation
}

\author{
Summaiya Iqbal' ${ }^{1}$ Paresh Mane ${ }^{2}$ and Dita Mayer ${ }^{1 *}$ \\ ${ }^{1}$ Department of Anesthesiology, Critical Care and Pain Medicine, USA \\ ${ }^{2}$ Department of Thoracic Surgery, St. Elizabeth's Medical Center, Tufts University School of Medicine, St Elizabeth's \\ Medical Center, USA
}

\begin{abstract}
Pigtail catheters are commonly used as effective alternative to traditional large bore chest tubes. However, the pigtail catheter placement does not come without possible complications. Pneumothorax, hemothorax and rarely visceral or vascular injury were all reported previously. Here we report a case of an 80 -year-old patient who presented with right-sided rib fractures and right hemothorax post mechanical fall. A pigtail drainage catheter (PC) was placed; however, minimal drainage was obtained and there was no resolution of hemothorax. CT evaluation showed the PC located between the liver and the underside of right hemidiaphragm. During the subsequent right video-assisted thoracoscopic surgery (VATS) the PC was found to be transthoracic and perforating the diaphragm but did not appear to enter the liver. We discuss the perioperative management of this patient as well as implications of possible PC complications for the anesthesiologists.
\end{abstract}

\section{Introduction}

In the setting of moderate-to-large size hemothorax, the tube thoracostomy is considered the primary intervention [1]. If drained early and adequately, the need for additional surgery is relatively rare (10-15\%) [2,3]. While large bore chest tubes are often preferred; multiple reports have suggested that smaller bore PC may be just as effective [4]. The smaller caliber is associated with less pain on insertion [5], while efficacy is preserved $[6,7]$. However, even smaller diameter PC placement does not come without possible complications, most commonly pneumothorax and hemothorax. Rare complications such as intraparenchymal insertion [8,9], left ventricular penetration [10], subclavian artery laceration and cerebral air embolism [11] have been reported previously. Proper training and imaging technique are required to perform pigtail catheter thoracostomy correctly and safely $[12,13]$.

\section{Case Report}

An 80-year-old male with history of type 2 diabetes, hypertension, and hyperlipidemia presented to our institution after a mechanical fall resulting in multiple right-sided rib fractures and right pleural effusion suspicious for hemothorax. The patient has sustained a mechanical fall when he slipped in his yard. He did not lose consciousness or suffered from any other injuries aside from as described below. Chest CT revealed a nondisplaced fracture of the left $11^{\text {th }}$ rib and the right posterior $12^{\text {th }}$ rib as well as was a slightly displaced and comminuted fracture of the right posterior $11^{\text {th }}$ rib and the right lateral $10^{\text {th }}$ rib. A moderate right pleural effusion was noted; however, flail chest was not present. Mild contusion in the right flank and lateral abdominal wall was observed. The patient did not sustain any other injuries. The patient was hemodynamically stable and not in respiratory distress. His $\mathrm{SpO}_{2}$ was $97 \%$ on room air did not require supplemental oxygen. He was not on any blood thinners and his coagulation parameters were within normal levels.

CT and x-ray images were reviewed, and a senior surgical resident attempted a chest tube insertion without ultrasound guidance. Multiple attempts at chest tube placement in the emergency room were unsuccessful (no blood was draining from the chest tube) and the patient was admitted to the hospital for further workup and hemothorax evacuation. Subsequently, a different surgical resident placed a pigtail drainage catheter (PC) without ultrasound guidance via the lateral right eighth intercostal space using Seldinger technique. Minimal drainage was obtained and there was no resolution of hemothorax. The lack of the catheter function prompted a CT evaluation and the PC appeared to be located

*Corresponding author: Dita Mayer, MD, Ph D, Department of Anesthesiology, Critical Care and Pain Medicine, St. Elizabeth's Medical Center, 736 Cambridge Street, CMP-2, Boston, MA 02135, USA Tel: +617-365-8154

Accepted: June 11, 2021

Published online: June 14, 2021

Citation: Iqbal S, Mane P, Mayer D (2021) A Case Report: TransThoracic Pigtail Catheter with Diaphragm Perforation. J Clin Anesth Pain Manag 5(1):224-226 
between the liver and the underside of right hemidiaphragm (Figure 1). There was no evidence of intraperitoneal bleeding; nevertheless, there was a concern for a possible liver injury.

The patient presented for right video-assisted thoracoscopic surgery for hemothorax evacuation and washout. Given the possibility of liver injury we have prepared for massive volume resuscitation that could result from the bleeding associated with the PC removal. A large bore i.v. (16G) and an a-line were placed pre induction and an additional i.v. (14G) was placed post induction. We had 2 units of pack red blood cells (PRBCs) in the operating room prior to incision and additional blood products were readily available in the blood bank. Belmont rapid transfuser (Belmont Medical Technologies, Billerica, MA) was primed and ready for use. FloTrac system (Edwards Life sciences Corp., Irivne, CA) was used to help manage possible hemodynamic instability.

During the surgery, the PC was found to be transthoracic and perforating the diaphragm (Figure 2) but did not appear to enter the liver. The source of hemothorax was $11^{\text {th }}$ rib fracture with a bleeding intercostal artery that was cauterized. The PC was then removed and there was no bleeding at the site of entry into the abdomen. The patient has recovered uneventfully and was discharged to a rehabilitation center on post-operative day five without any further complications.

\section{Discussion}

Pigtail drainage catheters while less invasive than traditional chest tubes do not come without complications

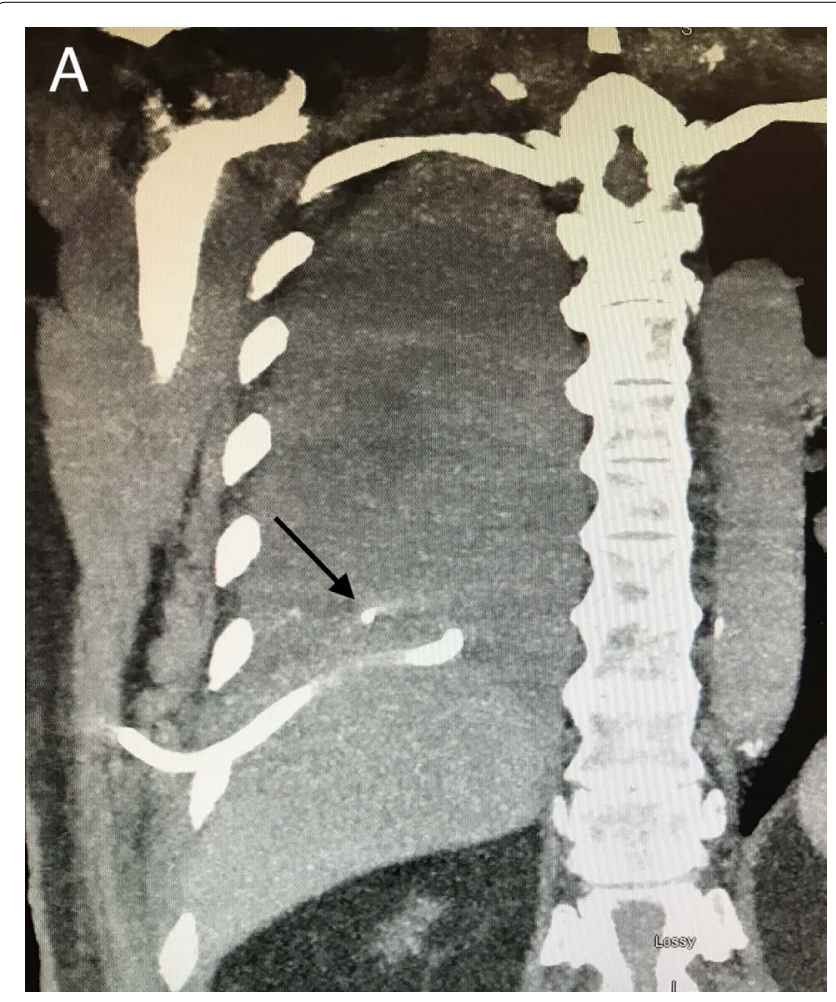

Figure 1: Computer tomography of chest and abdomen showing a pigtail drainage catheter (black arrow) placed via the lateral right eighth intercostal space and located between the liver and the underside of right hemidiaphragm. Furthermore, there is pneumothorax secondary to the catheter placement.

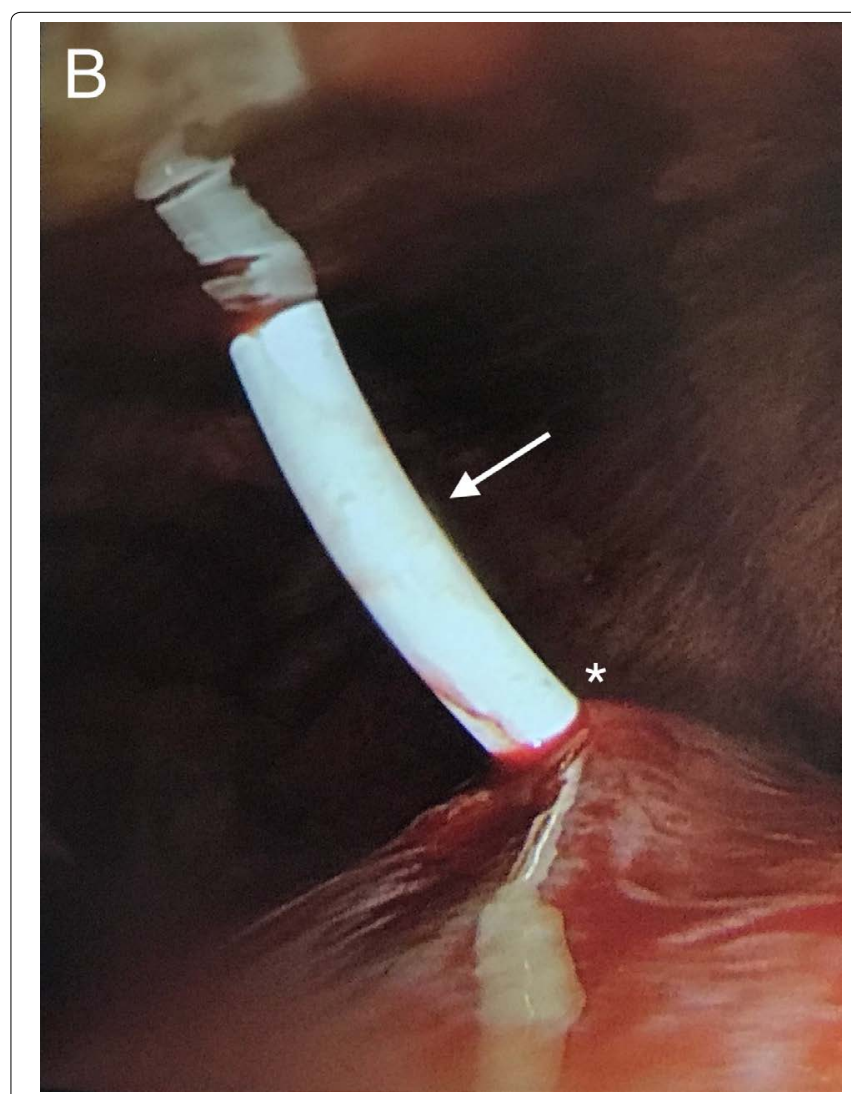

Figure 2: The pigtail catheter was found to be trans-thoracic (white arrow) and perforating the diaphragm (white asterisk) during the video-assisted thoracoscopic surgery.

$[6,7]$. The complications of chest pigtail catheters may include pain, pneumothorax, hemothorax, infection, dislodgement, kinking or blockage of the tube. Kinking and blockage occur more frequently with pigtail catheters given their smaller diameter [14]. In addition, rare complications such as air embolism [11,15], lung laceration $[9,10]$, and intrahepatic insertion [8] were described previously. Incidence of abdominal chest tube placement is about 1 to $7 \%[12,16]$. Proper procedure and technique reduce the likelihood of these complications. Intra-abdominal organ injury can be prevented by the use of a high approach and insertion of the pigtail drain catheter at or above the inter-mammary line in the fourth or fifth intercostal space [17]. Image guided technique, ideally ultrasound, should be utilized for pig tail insertion to minimize the risk of complications $[12,18]$. In addition, personnel involved with insertion of chest drains should be adequately trained and supervised $[12,18]$. In the case we present here, ultrasound guidance was not used, and the pigtail catheter was placed by a physician in training. It is possible that these factors contributed to the resulting malposition of the pigtail catheter.

Complications resulting from chest thoracostomy often necessitate emergent surgery. Anesthesiologists need to be aware of the complications related to the chest drain insertion to safely manage the patients in the operating room. Review of the available imaging, adequate intravenous access, hemodynamic monitoring as well as close communication with the surgical team are all required for the successful care of these patients. 


\section{Conclusion}

This case report emphasizes the importance of proper supervision and insertion technique for the chest drain placement. Complications resulting from misplaced chest drains can result in potentially life-threatening bleeding or organ injury. Anesthesiologists need to be aware of these complications and prepare accordingly.

\section{Declarations of Interest}

None.

\section{References}

1. Zeiler J, Idell S, Norwood S, et al. (2020) Hemothorax: A Review of the Literature. Clin Pulm Med 27: 1-12.

2. Goodman M, Lewis J, Guitron J, et al. (2013) Video-assisted thoracoscopic surgery for acute thoracic trauma. J Emerg Trauma Shock 6: 106-109.

3. Kong VY, Oosthuizen GV, Clarke DL (2015) Selective conservatism in the management of thoracic trauma remains appropriate in the 21st century. Ann R Coll Surg Engl 97: 224-228.

4. Bauman ZM, Kulvatunyou N, Joseph B, et al. (2018) A prospective study of 7-year experience using percutaneous 14-French pigtail catheters for traumatic hemothorax/hemopneumothorax at a level-1 trauma center: Size still does not matter. World J Surg 42: 107-113.

5. Kulvatunyou N, Erickson L, Vijayasekaran A, et al. (2014) Randomized clinical trial of pigtail catheter versus chest tube in injured patients with uncomplicated traumatic pneumothorax. Br J Surg 101: 17-22.

6. Gammie JS, Banks MC, Fuhrman CR, et al. (1999) The pigtail catheter for pleural drainage: A less invasive alternative to tube thoracostomy. JSLS 3: 57-61.
7. Orlando A, Cordero J, Carrick MM, et al. (2020) Comparing complications of small-bore chest tubes to large-bore chest tubes in the setting of delayed hemothorax: A retrospective multicenter cohort study. Scand J Trauma Resusc Emerg Med 28: 56.

8. Shen TC, Huang $\mathrm{KY}$, Chen $\mathrm{CH}$, et al. (2014) A rare complication of pig-tail catheter insertion. Am J Respir Crit Care Med 189: e64-e65.

9. Garcia de Alencar JC, Pinheiro Costa MG, Brandao Neto RA, et al. (2020) A Hidden Complication of Pigtail Catheter Insertion. Clin Pract Cases Emerg Med 4: 90-91.

10. Saqib A, Ibrahim U, Maroun R (2018) An unusual complication of pigtail catheter insertion. J Thorac Dis 10: 5964-5967.

11. Kim SI, Kwak HJ, Moon JY, et al. (2013) Cerebral air embolism following pigtail catheter insertion for pleural fluid drainage. Tuberc Respir Dis (Seoul) 74: 286-290.

12. Havelock T, Teoh R, Laws D, et al. (2010) Pleural procedures and thoracic ultrasound: British Thoracic Society Pleural Disease Guideline 2010. Thorax 65 Suppl 2: 61-76.

13. Molnar TF (2017) Thoracic Trauma: Which Chest Tube When and Where?. Thorac Surg Clin 27: 13-23.

14. Horsley A, Jones L, White J, et al. (2006) Efficacy and complications of small-bore, wire-guided chest drains. Chest 130: 1857-1863.

15. Alkhankan E, Nusair A, Mazagri R, et al. (2016) Systemic Air Embolism Associated with Pleural Pigtail Chest Tube Insertion. Case Rep Pulmonol 4053748.

16. Diacon $A H$, Brutsche $M H$, Soler $M$ (2003) Accuracy of pleural puncture sites: A prospective comparison of clinical examination with ultrasound. Chest 123: 436-441.

17. Poncia H, Ryan JM (2000) An unusual complication of chest tube thoracostomy. CJEM 2: 121-123.

18. Laws D, Neville E, Duffy J (2003) BTS guidelines for the insertion of a chest drain. Thorax 58: 53-59.

DOI: $10.36959 / 377 / 357$

Copyright: (c) 2021 lqbal S, et al. This is an open-access article distributed under the terms of the Creative Commons Attribution License, which permits unrestricted use, distribution, and reproduction in any medium, provided the original author and source are credited. 